East Asian Mathematical Journal Vol. 28 (2012), No. 5, pp. 597-604

http://dx.doi.org/10.7858/eamj.2012.045

\title{
VISCOSITY APPROXIMATION METHODS FOR NONEXPANSIVE SEMINGROUPS AND MONOTONE MAPPPINGS
}

\author{
LiJUAN ZHANG
}

\begin{abstract}
Let $C$ be a nonempty closed convex subset of real Hilbert space $H$ and $\mathfrak{F}=\{S(t): t \geq 0\}$ a nonexpansive self-mapping semigroup of $C$, and $f: C \rightarrow C$ is a fixed contractive mapping. Consider the process $\left\{x_{n}\right\}$ :

$$
\left\{\begin{array}{l}
x_{n+1}=\beta_{n} x_{n}+\left(1-\beta_{n}\right) z_{n} \\
z_{n}=\alpha_{n} f\left(x_{n}\right)+\left(1-\alpha_{n}\right) S\left(t_{n}\right) P_{C}\left(x_{n}-r_{n} A x_{n}\right) .
\end{array}\right.
$$

It is shown that $\left\{x_{n}\right\}$ converges strongly to a common element of the set of fixed points of nonexpansive semigroups and the set of solutions of the variational inequality for an inverse strongly-monotone mapping which solves some variational inequality.
\end{abstract}

\section{Introduction}

Let $H$ be a real Hilbert space with inner product $\langle\cdot, \cdot\rangle$ and induced norm $\|\cdot\|, C$ be a nonempty closed convex subset of $H$. $S: C \rightarrow C$ is nonexpansive if $\|S(x)-S(y)\| \leq\|x-y\|$, for all $x, y \in C$. The set of fixed points of $S$ is $F(S)=\{x \in C: x=S x\}$. We assume that $F(S) \neq \phi$, it is well known that $F(S)$ is closed convex.

A nonexpansive semigroup is a family $\mathfrak{F}=\{S(t): t \geq 0\}$ of self-mapping of $C$ if the following conditions are satisfied:

(a) $S(0) x=x$ for all $x \in C$;

(b) $S(s+t)=S(s) S(t)$ for all $s, t \geq 0$;

(c) For each $t>0,\|S(t) x-S(t) y\| \leq\|x-y\|, x, y \in C$.

(d) For each $x \in C$, the mapping $S(\cdot) x$ is continuous.

In this paper, we use $F$ to denote the set of common fixed points of $\mathfrak{F}$; that is,

$$
F=\{x \in C: S(t) x=x, t \geq 0\}=\bigcap_{t \geq 0} F(S(t)) .
$$

Received August 4, 2012; Accepted September 25, 2012.

2000 Mathematics Subject Classification. 47H09.

Key words and phrases. nonexpansive semigroup, viscosity approximation, inverse strongly monotone mapping, variational inequality, fixed point.

This work was financially supported by the NSF of Hebei province (A2012201054). 
The family $\{S(t): t \geq 0\}$ is said to be uniformly asymptotically regular if for any $t \geq 0$ and for any bounded subset $D$ of $C$,

$$
\lim _{s \rightarrow \infty} \sup _{x \in D}\|S(t+s) x-S(s) x\|=0 .
$$

A mapping $A: C \rightarrow H$ be a $\alpha$-inverse strongly mapping, if there exists a constant $\alpha>0$ such that

$$
\langle A x-A y, x-y\rangle \geq \alpha\|A x-A y\|^{2}, \forall x, y \in C
$$

then it is obvious that $A$ is $\frac{1}{\alpha}$-Lipschitz continuous. Recall that the classical variational inequality problem, denoted by $V I(C, A)$, is to find $x \in C$ such that

$$
\langle A x, y-x\rangle \geq 0, \forall y \in C .
$$

In 2001, Moudafi [2] introduced the viscosity approximation method for nonexpansive mappings. Let $f$ be a contraction on $C$, starting with an arbitrary initial $x_{0} \in C$, define a sequence $\left\{x_{n}\right\}$ recursively by

$$
x_{n+1}=\alpha_{n} f\left(x_{n}\right)+\left(1-\alpha_{n}\right) S x_{n}, n \geq 0 .
$$

In 2007, Song and Xu [4] introduced the iterative process given as follow:

$$
x_{n+1}=\alpha_{n} f\left(x_{n}\right)+\left(1-\alpha_{n}\right) S\left(t_{n}\right) x_{n}, n \geq 0 .
$$

They given an example of uniform asymptotically regular operator semigroup.

In this paper, motivated and inspired by the above results, we introduce an iterative scheme given as follows: $x_{1} \in C$ and

$$
\left\{\begin{array}{l}
x_{n+1}=\beta_{n} x_{n}+\left(1-\beta_{n}\right) z_{n} \\
z_{n}=\alpha_{n} f\left(x_{n}\right)+\left(1-\alpha_{n}\right) S\left(t_{n}\right) P_{C}\left(x_{n}-r_{n} A x_{n}\right) .
\end{array}\right.
$$

for all $n \in N$, where $\left\{\alpha_{n}\right\} \in[0,1]$ and $\left\{r_{n}\right\} \subset(0, \infty)$ satisfy some appropriate conditions. We will prove that $\left\{x_{n}\right\}$ converges strongly to $q \in F \cap V I(C, A)$.

\section{Preliminaries}

Let $H$ be a real Hilbert space with inner product $\langle\cdot, \cdot\rangle$ and norm $\|\cdot\|$, and let $C$ be a closed convex subset of $H$. We write $x_{n} \rightarrow x$ to indicate that the sequence $\left\{x_{n}\right\}$ converges weakly to $x . x_{n} \rightarrow x$ implies that $\left\{x_{n}\right\}$ converges strongly to $x$. For every point $x \in H$, there exists a unique nearest point in $C$, denoted by $P_{C} x$, such that

$$
\left\|x-P_{C} x\right\| \leq\|x-y\|
$$

for all $y \in C . P_{C}$ is called the metric projection of $H$ onto $C$. It is well known that $P_{C}$ satisfies:

$$
\left\langle x-y, P_{C} x-P_{C} y\right\rangle \geq\left\|P_{C} x-P_{C} y\right\|^{2}, \forall x, y \in H,
$$

and $P_{C}$ is characterized by the following properties:

$$
\begin{gathered}
\left\langle x-P_{C} x, P_{C} x-y\right\rangle \geq 0 \\
\|x-y\|^{2} \geq\left\|x-P_{C} x\right\|^{2}+\left\|y-P_{C} x\right\|^{2}, \forall x \in H, y \in C .
\end{gathered}
$$


In the context of the variational inequality problem, this implies

$$
u \in V I(C, A) \Leftrightarrow u=P_{C}(u-\lambda A u), \forall \lambda>0,
$$

We also have that for all $x, y \in C$ and $\lambda>0$,

$$
\begin{aligned}
& \|(I-\lambda A) x-(I-\lambda A) y\|^{2} \\
& =\|(x-y)-\lambda(A x-A y)\|^{2} \\
& =\|x-y\|^{2}-2 \lambda\langle x-y, A x-A y\rangle+\lambda^{2}\|A x-A y\|^{2} \\
& \leq\|x-y\|^{2}+\lambda(\lambda-2 \alpha)\|A x-A y\|^{2}
\end{aligned}
$$

So, if $\lambda \leq 2 \alpha$, then $I-\lambda A$ is a nonexpansive mapping of $C$ into $H$.

A set-valued mapping $T: H \rightarrow 2^{H}$ is called monotone if for all $x, y \in H, f \in$ $T x$ and $g \in T y$ imply $\langle x-y, f-g\rangle \geq 0$. A monotone mapping $T: H \rightarrow 2^{H}$ is maximal if graph $G(T)$ is not properly contained in the graph of any other monotone mapping. It is known that a monotone mapping $T$ is maximal if and only if for $(x, f) \in H \times H,\langle x-y, f-g\rangle \geq 0$ for every $(y, g) \in G(T)$ implies $f \in T x$. Let $A$ be an inverse-strongly monotone mapping of $C$ to $H$ and let $N_{C} v$ be normal cone to $C$ at $v \in C$, i.e.,

$$
N_{C} v=\{w \in H:\langle v-u, w\rangle \geq 0, \forall u \in C\}
$$

and define

$$
T v= \begin{cases}A v+N_{C} v, & v \in C, \\ \emptyset, & v \notin C .\end{cases}
$$

then $T$ is maximal monotone and $0 \in T v$ if and only if $v \in V I(C, A)$ [3].

Lemma 2.1. ([5]) Let $\left\{x_{n}\right\},\left\{y_{n}\right\}$ be bounded sequences in a Banach space $E$ and $\left\{\beta_{n}\right\}$ be a sequence in $[0,1]$ with $0<\liminf \beta_{n} \leq \lim \sup \beta_{n}<1$, Suppose $x_{n+1}=\beta_{n} y_{n}+\left(1-\beta_{n}\right) x_{n}, \forall n \geq 0$ and $\lim \sup _{n \rightarrow \infty}\left(\left\|y_{n+1}-y_{n}\right\|-\left\|x_{n+1}-x_{n}\right\|\right) \leq$ 0. Then $\lim _{n \rightarrow \infty}\left\|y_{n}-x_{n}\right\|=0$.

Lemma 2.2. ([6]) Let $\left\{s_{n}\right\}$ be a sequence of nonnegative real numbers such that:

$$
s_{n+1} \leq\left(1-\lambda_{n}\right) s_{n}+\beta_{n}, n \geq 0
$$

where $\left\{\lambda_{n}\right\},\left\{\beta_{n}\right\}$ satisfy the conditions:

(i) $\left\{\lambda_{n}\right\} \subset(0,1)$ and $\sum_{n=1}^{\infty} \lambda_{n}=\infty$,

(ii) $\limsup _{n \rightarrow \infty} \frac{\beta_{n}}{\lambda_{n}} \leq 0$ or $\sum_{n=1}^{\infty}\left|\beta_{n}\right|<\infty$. Then $\lim _{n \rightarrow \infty} s_{n}=0$.

Lemma 2.3. ([1]) Let $C$ a nonempty bounded closed convex subset of $H$ and $\{S(t): t \geq 0\}$ be a nonexpansive semigroup on $C$. If $\left\{x_{n}\right\}$ is a sequence in $C$ such that $x_{n} \rightarrow z$ and $\lim \sup _{t} \lim \sup _{n}\left\|x_{n}-S(t) x_{n}\right\| \rightarrow 0$, then $z \in F(S)$. 


\section{Main result}

Theorem 3.1. Let $H$ be a real Hilbert space, and $C$ be a nonempty closed convex subset of $H$. Let $\{S(t): t \geq 0\}$ be a uniform asymptotically regular nonexpansive semigroup from $C$ to $C$, and $A$ an $\alpha$-inverse-strongly monotone mapping of $C$ into $H$ such that $F \cap V I(C, A) \neq \emptyset . f: C \rightarrow C$ is a contraction with coefficient $k \in(0,1)$, Suppose $\left\{x_{n}\right\}$ be sequences generated by (1.1), where $\left\{\alpha_{n}\right\}$ and $\left\{\beta_{n}\right\}$ is sequence in $(0,1)$ and $\lim _{n \rightarrow \infty} t_{n}=\infty$. If $\left\{r_{n}\right\}$ are chosen so that $r_{n} \in[a, b]$ for some $a, b$ with $0<a<b<2 \alpha$,

$\lim _{n \rightarrow \infty} \alpha_{n}=0, \sum_{n=1}^{\infty} \alpha_{n}=\infty, \quad \lim _{n \rightarrow \infty}\left|r_{n}-r_{n+1}\right|=0, \quad 0<\liminf _{n \rightarrow \infty} \beta_{n} \leq \limsup _{n \rightarrow \infty} \beta_{n}<1$.

Then $\left\{x_{n}\right\}$ converges strongly to $q \in F \cap V I(C, A)$, which is the unique solution in the $F \cap V I(C, A)$ to the following variational inequality

$$
\langle f(q)-q, p-q\rangle \leq 0, \quad \forall p \in F \cap V I(C, A) .
$$

Proof. It is easy to see that the uniqueness of a solution of the above variational inequality. Since $I-f$ is strongly monotone, so the variational inequality has only one solution.

Put $y_{n}=P_{C}\left(x_{n}-r_{n} A x_{n}\right)$ for every $n=0,1,2, \ldots$ Let $p \in F \cap V I(C, A)$. Notice that

$$
\begin{aligned}
\left\|y_{n}-p\right\| & =\left\|P_{C}\left(x_{n}-r_{n} A x_{n}\right)-P_{C}\left(p-r_{n} A p\right)\right\| \\
& \leq\left\|x_{n}-r_{n} A x_{n}-\left(p-r_{n} A p\right)\right\| \\
& \leq\left\|x_{n}-p\right\| .
\end{aligned}
$$

Then we compute that

$$
\begin{aligned}
\left\|z_{n}-p\right\| & \leq \alpha_{n}\left\|f\left(x_{n}\right)-p\right\|+\left(1-\alpha_{n}\right)\left\|S\left(t_{n}\right) y_{n}-p\right\| \\
& \leq \alpha_{n}\left\|f\left(x_{n}\right)-f(p)\right\|+\alpha_{n}\|f(p)-p\|+\left(1-\alpha_{n}\right)\left\|y_{n}-p\right\| \\
& \leq \alpha_{n} k\left\|x_{n}-p\right\|+\alpha_{n}\|f(p)-p\|+\left(1-\alpha_{n}\right)\left\|x_{n}-p\right\| \\
& =\left[1-\alpha_{n}(1-k)\right]\left\|x_{n}-p\right\|+\alpha_{n}\|f(p)-p\| .
\end{aligned}
$$

so we have

$$
\begin{aligned}
\left\|x_{n+1}-p\right\| \leq & \beta_{n}\left\|x_{n}-p\right\|+\left(1-\beta_{n}\right)\left\|z_{n}-p\right\| \\
\leq & \beta_{n}\left\|x_{n}-p\right\|+\left(1-\beta_{n}\right)\left[1-\alpha_{n}(1-k)\right]\left\|x_{n}-p\right\| \\
& +\left(1-\beta_{n}\right) \alpha_{n}\|f(p)-p\| \\
\leq & {\left[1-\left(1-\beta_{n}\right) \alpha_{n}(1-k)\right]\left\|x_{n}-p\right\|+\left(1-\beta_{n}\right) \alpha_{n}\|f(p)-p\| . }
\end{aligned}
$$

By induction

$$
\left\|x_{n}-p\right\| \leq \max \left\{\left\|x_{0}-p\right\|, \frac{1}{1-k}\|f(p)-p\|\right\}, n \geq 0 .
$$

Therefore $\left\{x_{n}\right\}$ is bounded, we have $\left\{z_{n}\right\},\left\{y_{n}\right\},\left\{S\left(t_{n}\right) y_{n}\right\},\left\{A x_{n}\right\},\left\{f\left(x_{n}\right)\right\}$ 
are also bounded. Since $I-r A$ is nonexpansive, we have

$$
\begin{aligned}
\left\|y_{n+1}-y_{n}\right\| & \leq\left\|x_{n+1}-r_{n+1} A x_{n+1}-\left(x_{n}-r_{n} A x_{n}\right)\right\| \\
& \leq\left\|x_{n+1}-r_{n+1} A x_{n+1}-\left(x_{n}-r_{n+1} A x_{n}\right)\right\|+\left|r_{n}-r_{n+1}\right|\left\|A x_{n}\right\| \\
& \leq\left\|x_{n+1}-x_{n}\right\|+\left|r_{n}-r_{n+1}\right|\left\|A x_{n}\right\| .
\end{aligned}
$$

So we obtain (for some appropriate constant $M>0$ )

$$
\begin{aligned}
\left\|z_{n+1}-z_{n}\right\| \leq & \alpha_{n+1}\left\|f\left(x_{n+1}\right)-f\left(x_{n}\right)\right\|+\left|\alpha_{n+1}-\alpha_{n}\right|\left\|f\left(x_{n}\right)-S\left(t_{n+1}\right) y_{n}\right\| \\
& +\left(1-\alpha_{n+1}\right)\left\|S\left(t_{n+1}\right)\left(y_{n+1}-y_{n}\right)\right\| \\
& +\left(1-\alpha_{n}\right)\left\|S\left(t_{n+1}\right) y_{n}-S\left(t_{n}\right) y_{n}\right\| \\
\leq & \alpha_{n+1} k\left\|x_{n+1}-x_{n}\right\|+\left|\alpha_{n}-\alpha_{n+1}\right| M+\left(1-\alpha_{n+1}\right)\left\|y_{n+1}-y_{n}\right\| \\
& +\left(1-\alpha_{n}\right)\left\|S\left(t_{n+1}\right) y_{n}-S\left(t_{n}\right) y_{n}\right\| \\
\leq & \left(1-\alpha_{n+1}+\alpha_{n+1} k\right)\left\|x_{n+1}-x_{n}\right\|+\left|\alpha_{n}-\alpha_{n+1}\right| M \\
& +\left|r_{n}-r_{n+1}\right| M+\left(1-\alpha_{n}\right)\left\|S\left(t_{n+1}\right) y_{n}-S\left(t_{n}\right) y_{n}\right\|
\end{aligned}
$$

So we have

$$
\begin{aligned}
& \left\|z_{n+1}-z_{n}\right\|-\left\|x_{n+1}-x_{n}\right\| \\
& \leq\left(\alpha_{n+1} k-\alpha_{n+1}\right)\left\|x_{n+1}-x_{n}\right\|+\left|\alpha_{n}-\alpha_{n+1}\right| M \\
& \quad+\left|r_{n}-r_{n+1}\right| M+\left(1-\alpha_{n}\right)\left\|S\left(\left(t_{n+1}-t_{n}\right)+t_{n}\right) y_{n}-S\left(t_{n}\right) y_{n}\right\| \\
& \leq\left(\alpha_{n+1} k-\alpha_{n+1}\right)\left\|x_{n+1}-x_{n}\right\|+\left|\alpha_{n}-\alpha_{n+1}\right| M \\
& \quad+\left|r_{n}-r_{n+1}\right| M+\left(1-\alpha_{n}\right) \sup _{y \in\left\{y_{n}\right\}, t \geq 0}\left\|S\left(t+t_{n}\right) y-S\left(t_{n}\right) y\right\|
\end{aligned}
$$

Since $\lim _{n \rightarrow \infty} \alpha_{n}=0$ and $\lim _{n \rightarrow \infty}\left|r_{n}-r_{n+1}\right|=0$ and the uniform asymptotic regularity of the nonexpansive semigroup, we have

$$
\limsup _{n \rightarrow \infty}\left(\left\|z_{n+1}-z_{n}\right\|-\left\|x_{n+1}-x_{n}\right\|\right)=0
$$

In view of Lemma 2.1, we have $\lim _{n \rightarrow \infty}\left\|z_{n+1}-x_{n}\right\|=0,\left\|x_{n+1}-x_{n}\right\| \rightarrow 0$ and $\left\|y_{n+1}-y_{n}\right\| \rightarrow 0$. From the definition of $x_{n}$, we have

$$
\begin{aligned}
\left\|z_{n}-p\right\|^{2} & \leq \alpha_{n}\left\|f\left(x_{n}\right)-p\right\|^{2}+\left(1-\alpha_{n}\right)\left\|S\left(t_{n}\right) y_{n}-p\right\|^{2} \\
& \leq \alpha_{n}\left\|f\left(x_{n}\right)-p\right\|^{2}+\left(1-\alpha_{n}\right)\left\|y_{n}-p\right\|^{2} \\
& \leq \alpha_{n}\left\|f\left(x_{n}\right)-p\right\|^{2}+\left(1-\alpha_{n}\right)\left[\left\|x_{n}-p\right\|^{2}+r_{n}\left(r_{n}-2 \alpha\right)\left\|A x_{n}-A p\right\|^{2}\right] \\
& \leq \alpha_{n}\left\|f\left(x_{n}\right)-p\right\|^{2}+\left\|x_{n}-p\right\|^{2}+\left(1-\alpha_{n}\right) a(b-2 \alpha)\left\|A x_{n}-A p\right\|^{2}
\end{aligned}
$$

For $p \in F \cap V I(C, A)$, we have

$$
\begin{aligned}
\left\|x_{n+1}-p\right\|^{2} \leq & \beta_{n}\left\|x_{n}-p\right\|^{2}+\left(1-\beta_{n}\right)\left\|z_{n}-p\right\|^{2} \\
\leq & \beta_{n}\left\|x_{n}-p\right\|^{2}+\left(1-\beta_{n}\right)\left[\alpha_{n}\left\|f\left(x_{n}\right)-p\right\|^{2}+\left\|x_{n}-p\right\|^{2}\right. \\
& \left.+\left(1-\alpha_{n}\right) a(b-2 \alpha)\left\|A x_{n}-A p\right\|^{2}\right] \\
\leq & \left\|x_{n}-p\right\|^{2}+\left(1-\alpha_{n}\right)\left(1-\beta_{n}\right) a(b-2 \alpha)\left\|A x_{n}-A p\right\|^{2} \\
& +\alpha_{n}\left\|f\left(x_{n}\right)-p\right\|^{2}
\end{aligned}
$$


So, we obtain

$$
\begin{aligned}
& \left(1-\alpha_{n}\right)\left(1-\beta_{n}\right) a(2 \alpha-b)\left\|A x_{n}-A p\right\|^{2} \\
& \leq \alpha_{n}\left\|f\left(x_{n}\right)-p\right\|^{2}+\left(\left\|x_{n+1}-p\right\|+\left\|x_{n}-p\right\|\right)\left\|x_{n}-x_{n+1}\right\| .
\end{aligned}
$$

Since $0<\liminf _{n \rightarrow \infty} \beta_{n} \leq \limsup _{n \rightarrow \infty} \beta_{n}<1, \alpha_{n} \rightarrow 0$ and $\left\|x_{n}-x_{n+1}\right\| \rightarrow 0$, then $\left\|A x_{n}-A p\right\| \rightarrow 0, n \rightarrow \infty$.

Further, from (2.1) we have

$$
\begin{aligned}
\left\|y_{n}-p\right\|^{2}= & \left\|P_{C}\left(x_{n}-r_{n} A x_{n}\right)-P_{C}\left(p-r_{n} A p\right)\right\|^{2} \\
\leq & \left\langle x_{n}-r_{n} A x_{n}-\left(p-r_{n} A p\right), y_{n}-p\right\rangle \\
= & \frac{1}{2}\left\{\|\left(x_{n}-r_{n} A x_{n}-\left(p-r_{n} A p\right)\left\|^{2}+\right\| y_{n}-p \|^{2}\right.\right. \\
& \left.-\left\|x_{n}-r_{n} A x_{n}-\left(p-r_{n} A p\right)-\left(y_{n}-p\right)\right\|^{2}\right\} \\
\leq & \frac{1}{2}\left\{\left\|x_{n}-p\right\|^{2}+\left\|y_{n}-p\right\|^{2}-\left\|x_{n}-y_{n}-r_{n}\left(A x_{n}-A p\right)\right\|^{2}\right\} \\
= & \frac{1}{2}\left\{\left\|x_{n}-p\right\|^{2}+\left\|y_{n}-p\right\|^{2}-\left\|x_{n}-y_{n}\right\|^{2}\right. \\
& \left.+2 r_{n}\left\langle x_{n}-y_{n}, A x_{n}-A p\right\rangle-r_{n}^{2}\left\|A x_{n}-A p\right\|^{2}\right\} .
\end{aligned}
$$

This implies that

$$
\begin{aligned}
\left\|y_{n}-p\right\|^{2} \leq & \left\|x_{n}-p\right\|^{2}-\left\|x_{n}-y_{n}\right\|^{2}+2 r_{n}\left\langle x_{n}-y_{n}, A x_{n}-A p\right\rangle \\
& -r_{n}^{2}\left\|A x_{n}-A p\right\|^{2} \\
\leq & \left\|x_{n}-p\right\|^{2}-\left\|x_{n}-y_{n}\right\|^{2}+2 r_{n}\left\|x_{n}-y_{n}\right\|\left\|A x_{n}-A p\right\| .
\end{aligned}
$$

So we have

$$
\begin{aligned}
\left\|x_{n+1}-p\right\|^{2} \leq & \beta_{n}\left\|x_{n}-p\right\|^{2}+\left(1-\beta_{n}\right)\left\|z_{n}-p\right\|^{2} \\
\leq & \beta_{n}\left\|x_{n}-p\right\|^{2}+\left(1-\beta_{n}\right)\left[\alpha_{n}\left\|f\left(x_{n}\right)-p\right\|^{2}\right. \\
& \left.+\left(1-\alpha_{n}\right)\left\|S\left(t_{n}\right) y_{n}-p\right\|^{2}\right] \\
\leq & \beta_{n}\left\|x_{n}-p\right\|^{2}+\left(1-\beta_{n}\right)\left\|y_{n}-p\right\|^{2}+\alpha_{n}\left\|f\left(x_{n}\right)-p\right\|^{2} \\
\leq & \alpha_{n}\left\|f\left(x_{n}\right)-p\right\|^{2}+\left\|x_{n}-p\right\|^{2}-\left(1-\beta_{n}\right)\left\|x_{n}-y_{n}\right\|^{2} \\
& +2 r_{n}\left\|x_{n}-y_{n}\right\|\left\|A x_{n}-A p\right\| .
\end{aligned}
$$

which implies that

$$
\begin{aligned}
\left(1-\beta_{n}\right)\left\|x_{n}-y_{n}\right\|^{2} \leq & 2 r_{n}\left\|x_{n}-y_{n}\right\|\left\|A x_{n}-A p\right\|+\alpha_{n}\left\|f\left(x_{n}\right)-p\right\|^{2} \\
& +\left\|x_{n}-p\right\|^{2}-\left\|x_{n+1}-p\right\|^{2}
\end{aligned}
$$

Since $\alpha_{n} \rightarrow 0,0<\liminf _{n \rightarrow \infty} \beta_{n} \leq \limsup _{n \rightarrow \infty} \beta_{n}<1,\left\|x_{n}-x_{n+1}\right\| \rightarrow 0$ and $\left\|A x_{n}-A p\right\| \rightarrow 0$, we have $\left\|x_{n}-y_{n}\right\| \rightarrow 0$. Since

$$
\begin{aligned}
\left\|x_{n}-S\left(t_{n}\right) x_{n}\right\| & \leq\left\|x_{n}-z_{n}\right\|+\left\|z_{n}-S\left(t_{n}\right) y_{n}\right\|+\left\|S\left(t_{n}\right) y_{n}-S\left(t_{n}\right) x_{n}\right\| \\
& \leq\left\|x_{n}-z_{n}\right\|+\alpha_{n}\left\|f\left(x_{n}\right)-S\left(t_{n}\right) y_{n}\right\|+\left\|y_{n}-x_{n}\right\| .
\end{aligned}
$$


From $\alpha_{n} \rightarrow 0$, we have $\lim _{n \rightarrow \infty}\left\|x_{n}-S\left(t_{n}\right) x_{n}\right\|=0$. Further

$$
\begin{aligned}
\left\|x_{n}-S(t) x_{n}\right\| \leq & \left\|x_{n}-S\left(t_{n}\right) x_{n}\right\|+\left\|S\left(t_{n}\right) x_{n}-S(t) S\left(t_{n}\right) x_{n}\right\| \\
& +\left\|S(t) S\left(t_{n}\right) x_{n}-S(t) x_{n}\right\| \\
\leq & 2\left\|x_{n}-S\left(t_{n}\right) x_{n}\right\|+\sup _{x \in\left\{x_{n}\right\}, t \geq 0}\left\|S\left(t+t_{n}\right) x-S\left(t_{n}\right) x\right\| .
\end{aligned}
$$

Using this and the uniform asymptotic regularity of the nonexpansive semigroup, we get

$$
\left\|x_{n}-S(t) x_{n}\right\| \rightarrow 0, \forall t \geq 0 .
$$

Choose a subsequence $\left\{y_{n_{i}}\right\}$ of $\left\{y_{n}\right\}$ such that

$$
\limsup _{n \rightarrow \infty}\left\langle f(q)-q, S\left(t_{n}\right) y_{n}-q\right\rangle=\lim _{i \rightarrow \infty}\left\langle f(q)-q, S\left(t_{n_{i}}\right) y_{n_{i}}-q\right\rangle .
$$

As $\left\{y_{n_{i}}\right\}$ is bounded, Without loss of generality that $y_{n_{i}} \rightarrow z \in C$. Since $\left\|S\left(t_{n}\right) y_{n}-y_{n}\right\| \rightarrow 0$, we obtain $S\left(t_{n_{i}}\right) y_{n_{i}} \rightarrow z \in C$. In fact, Let

$$
T v= \begin{cases}A v+N_{C} v, & v \in C, \\ \emptyset, & v \notin C .\end{cases}
$$

Then $T$ is maximal monotone. Let $(v, w) \in G(T)$. Since $w-A v \in N_{C} v$ and $y_{n} \in C$ we have

$$
\left\langle v-y_{n}, w-A v\right\rangle \geq 0 .
$$

On the other hand, from $y_{n}=P_{C}\left(x_{n}-r_{n} A x_{n}\right)$, we have $\left\langle v-y_{n}, y_{n}-\left(x_{n}-\right.\right.$ $\left.\left.r_{n} A x_{n}\right)\right\rangle \geq 0$ and hence

$$
\left\langle v-y_{n}, \frac{y_{n}-x_{n}}{r_{n}}+A x_{n}\right\rangle \geq 0
$$

Therefore, we have

$$
\begin{aligned}
\left\langle v-y_{n_{i}}, w\right\rangle \geq & \left\langle v-y_{n_{i}}, A v\right\rangle \\
\geq & \left\langle v-y_{n_{i}}, A v\right\rangle-\left\langle v-y_{n_{i}}, \frac{y_{n_{i}}-x_{n_{i}}}{r_{n_{n}}}+A x_{n_{i}}\right\rangle \\
= & \left\langle v-y_{n_{i}}, A v-A y_{n_{i}}\right\rangle+\left\langle v-y_{n_{i}}, A y_{n_{i}}-A x_{n_{i}}\right\rangle \\
& -\left\langle v-y_{n_{i}}, \frac{y_{n_{i}}-x_{n_{i}}}{r_{n_{i}}}\right\rangle \\
\geq & \left\langle v-y_{n_{i}}, A y_{n_{i}}-A x_{n_{i}}\right\rangle-\left\langle v-y_{n_{i}}, \frac{y_{n_{i}}-x_{n_{i}}}{r_{n_{i}}}\right\rangle
\end{aligned}
$$

Hence we have $\langle v-z, w\rangle \geq 0$ as $i \rightarrow \infty$. Since $T$ is maximal monotone, we have $z \in T^{-1} 0$ and $z \in V I(C, A)$. From (3.1) and Lemma 2.3, we have $z \in F$.

Further we have

$$
\begin{aligned}
\limsup _{n \rightarrow \infty}\left\langle f(q)-q, S\left(t_{n}\right) y_{n}-q\right\rangle & =\lim _{i \rightarrow \infty}\left\langle f(q)-q, S\left(t_{n_{i}}\right) y_{n_{i}}-q\right\rangle \\
& =\langle f(q)-q, z-q\rangle \leq 0
\end{aligned}
$$


We compute that

$$
\begin{aligned}
\left\|x_{n+1}-q\right\|^{2} \leq & \beta_{n}\left\|x_{n}-q\right\|^{2}+\left(1-\beta_{n}\left[2 \alpha_{n}\left(1-\alpha_{n}\right)\left\langle f\left(x_{n}\right)-q, S\left(t_{n}\right) y_{n}-q\right\rangle\right.\right. \\
& \left.+\alpha_{n}^{2}\left\|f\left(x_{n}\right)-q\right\|^{2}+\left(1-\alpha_{n}\right)^{2}\left\|S\left(t_{n}\right) y_{n}-q\right\|^{2}\right] \\
\leq & \beta_{n}\left\|x_{n}-q\right\|^{2}+2\left(1-\beta_{n}\right) \alpha_{n}\left(1-\alpha_{n}\right)\left\langle f\left(x_{n}\right)-f(q), S\left(t_{n}\right) y_{n}-q\right\rangle \\
& +2\left(1-\beta_{n}\right) \alpha_{n}\left(1-\alpha_{n}\right)\left\langle f(q)-q, S\left(t_{n}\right) y_{n}-q\right\rangle \\
& +\left(1-\beta_{n}\right) \alpha_{n}^{2}\left\|f\left(x_{n}\right)-q\right\|^{2}+\left(1-\beta_{n}\right)\left(1-\alpha_{n}\right)^{2}\left\|y_{n}-q\right\|^{2} \\
\leq & \beta_{n}\left\|x_{n}-q\right\|^{2}+2 k\left(1-\beta_{n}\right) \alpha_{n}\left(1-\alpha_{n}\right)\left\|x_{n}-q\right\|^{2} \\
& +2\left(1-\beta_{n}\right) \alpha_{n}\left(1-\alpha_{n}\right)\left\langle f(q)-q, S\left(t_{n}\right) y_{n}-q\right\rangle \\
& +\left(1-\beta_{n}\right) \alpha_{n}^{2}\left\|f\left(x_{n}\right)-q\right\|^{2}+\left(1-\beta_{n}\right)\left(1-\alpha_{n}\right)^{2}\left\|x_{n}-q\right\|^{2} \\
\leq & \left\{1-\left(1-\beta_{n}\right) \alpha_{n}\left[2-\alpha_{n}-2 k\left(1-\alpha_{n}\right)\right]\right\}\left\|x_{n}-q\right\|^{2} \\
& +\left(1-\beta_{n}\right) \alpha_{n}^{2}\left\|f\left(x_{n}\right)-q\right\|^{2} \\
& +2\left(1-\beta_{n}\right) \alpha_{n}\left(1-\alpha_{n}\right)\left\langle f(q)-q, S\left(t_{n}\right) y_{n}-q\right\rangle \\
= & \left(1-\overline{\alpha_{n}}\right)\left\|x_{n}-q\right\|^{2}+\overline{\alpha_{n}} \overline{\beta_{n} .}
\end{aligned}
$$

where

$$
\begin{aligned}
\overline{\alpha_{n}} & =\left(1-\beta_{n}\right) \alpha_{n}\left[2-\alpha_{n}-2 k\left(1-\alpha_{n}\right)\right], \\
\overline{\beta_{n}} & =\frac{\alpha_{n}\left\|f\left(x_{n}\right)-q\right\|^{2}+\left(1-\alpha_{n}\right)\left\langle f(q)-q, S\left(t_{n}\right) y_{n}-q\right\rangle}{2-\alpha_{n}-2 k\left(1-\alpha_{n}\right)} .
\end{aligned}
$$

It is easily seen that $\overline{\alpha_{n}} \rightarrow 0, \sum_{n=1}^{\infty} \overline{\alpha_{n}}=\infty$, and $\lim \sup _{n \rightarrow \infty} \overline{\beta_{n}} \leq 0$, by Lemma 2.2, we obtain $x_{n} \rightarrow q$.

\section{References}

[1] T. H. Kim and H. K. Xu, Strong convergence of modified Mann iteration for asymptotically nonexpansive mappings and semigroups, Nonliear Anal. 64 (2006), 1140-1152.

[2] A. Moudafi, Viscosity approximation methods for fixed points problems, J. Math. Anal. Appl. 241 (2000), 46-55.

[3] R. T. Rockafellar, On the Maximality of Sums of Nonlinear Monotone Operators, Transactions of the American Mathematical Society 149 (1970), 75-88.

[4] Y. Song and S. Xu, Strong convergence theorems for nonexpansive semigroup in Banach spaces, J. Math. Anal. Appl. 318 (2007), 43-52.

[5] T. Suzuki, Strong convergence of Krasnoselskii and Mann's type sequences for one parameter nonexpansive semigroups without Bochner integrals, J. Math. Anal. Appl. 305 (2005), 227-239.

[6] H. K. Xu, An iterative approach to quadratic optimization, J. Optim. Theory Appl. 116 (2003), 659-678.

LIJUAN ZHANG

College of Mathematics and Computer, Hebei University, Baoding, 071002, PR CHINA

E-mail address: zhanglj@hbu.edu.cn 